\title{
Data Driven Fault Detection and Isolation of a Wind Turbine Benchmark
}

\author{
Jianfei Dong* Michel Verhaegen* \\ ${ }^{*}$ Delft University of Technology, 2628CD, Delft, the Netherlands. \\ Email: j.dong@tudelft.nl, M.Verhaegen@moesp.org
}

\begin{abstract}
This paper investigates data-driven fault detection and isolation (FDI) designs for a wind turbine benchmark problem. The benchmark is described by a SimuLink model, which contains nonlinear lookup tables and unknown wind disturbances. Based on classical FDI design methods, a linearization of the SimuLink model into a standard state-space form and describing the linearization errors as perturbations may be necessary. To avoid these difficult modeling procedures, this paper applies a data-driven design method, which produces FDI filters directly based on the simulated data from the benchmark SimuLink model. The fixed-value sensor faults therein are especially targeted. Moreover, we develop in this paper a new data-driven fault isolation scheme, via exploiting hardware redundancy in a plant. Based on this, a bank of robust data-driven detection filters are designed for the benchmark and implemented in parallel. The simulation results show the effectiveness of the applied data-driven scheme.
\end{abstract}

Keywords: Fault detection and isolation; Wind turbines; Data-driven methods; Statistical analysis; Subspace identification.

\section{INTRODUCTION}

Generating electricity from wind has become a reality in recent years. However, potential faults of wind turbines can occur in their gearbox, blades, sensors, the motors yawing the rotor, and etc. These faults cause remarkable downtime. The maintenance of a wind turbine, where these faults have occurred, is actually a big challenge for industrial practitioners, especially for offshore large-scale wind turbines. The challenge can actually be attributed to the difficulty and even the danger in accessing the turbines. This hence necessitates the availability of automatic detection and isolation of the faults and reconfiguration of the control system accordingly. Driven by this need, research in FDI design for wind turbines has been recently been carried out in literature, e.g. [Wei et al., 2010]. Moreover, a benchmark problem has been defined in [Odgaard et al., 2009].

In the existing literature, FDI of wind turbines has been developed based on signal processing and artificial intelligence (AI) techniques, e.g. [Caselitz et al., 1997]. Signal processing is to extract features from measured data; while AI is used to classify the features to different conditions. This is also known as a "black-box" approach. However, black-box approaches usually suffer from lacking the robustness against unseen data. Whereas, for a dynamic process, the initial state and control signals determine the readings from sensors. Black-box methods cope with these variations by performing as many experiments as possible. An alternative way is of course to rely on the dynamic model of a wind turbine, which take the initial state and control signals into account. This is the so-called "modelbased" approaches, e.g. [Wei and Verhaegen, 2008, Wei et al., 2010].
As model based FDI becoming a well-established literature [Basseville and Nikiforov, 1993, Chen and Patton, 1999, Ding, 2008, Gustafsson, 2001], numerous tools have been developed. With the help of these tools, the main efforts in implementing an FDI scheme on a wind turbine is not in developing the algorithms themselves, but in modeling the dynamics. Modeling by first principles is what users can rely on. However, modeling a wind turbine is a rather difficult task [van Engelen, 2007]. Besides, a good simulation model may not be as good for FDI design. For instance, the benchmark model in [Odgaard et al., 2009] contains lookup tables, which defines complicated nonlinear mappings, and can only be approximated by continuous functions. The difficulties in modeling have stimulated the development of data-driven FDI methods in literature [Dong et al., 2009, 2010, 2011, Qin and Li, 2001, Ding et al., 2009]. Based on subspace identification methods [Verhaegen and Verdult, 2007], the data-driven PSA approaches in [Qin and Li, 2001, Ding et al., 2009] require computing the left null space of the identified range space of the extended observability matrix, directly identified from measurement data, without realizing the state-space matrices, e.g. $(A, B, C, D)$. In [Dong et al., 2009, 2010, 2011], a method called $F$ d $I$ scheme Connected to Subspace Identification (FICSI) has been developed to avoid the projection of residual vectors as in PSA. The benefits of avoiding the projection include the easily identifiable FDI filter parameters as a single least-squares problem, and the explicit quantification of the parameter uncertainties on the residual distribution.

In addition to the nonlinearity in its simulation model, the power of the measurement noise in all the sensor signals defined in [Odgaard et al., 2009] is high. These factors make the FDI problem very challenging. In this current 
paper, we intend to apply the data-driven FICSI design of Dong et al. [2010, 2011] to especially handle the fixed-value sensor faults therein with robustness to the stochastic parameter errors induced by the low SNR. We will also develop a fault isolation scheme for this design.

The rest of the paper is organized as follows. Section 2 reviews the data-driven FICSI approach and its robust solution. Section 3 develops a fault isolation scheme based on hardware redundancy for the robustified data-driven FICSI detection method. The wind turbine benchmark problem is then described in Section 4, together with the FDI results of the fixed-value sensor faults therein. Section 5 finally concludes this paper.

\section{ROBUST DATA-DRIVEN FAULT DETECTION DESIGN}

\subsection{Nominal data-driven solution}

In modern computer controlled systems, a model usually takes the following discrete-time state space form.

$$
\begin{aligned}
x(k+1) & =A x(k)+B u(k)+F w(k), \\
y(k) & =C x(k)+D u(k)+v(k) .
\end{aligned}
$$

Here, we consider MIMO systems; i.e. $x(k) \in \mathbb{R}^{n}, y(k) \in$ $\mathbb{R}^{\ell}$, and $u(k) \in \mathbb{R}^{m}$. A, B,C,D,F are real bounded matrices with appropriate dimensions. The disturbances are represented by the process noise $w(k) \in \mathbb{R}^{n_{w}}$ and the measurement noise $v(k) \in \mathbb{R}^{n_{v}}$. As standard assumptions in system identification, the measurement and the process noise, $w(k), v(k)$, are white zero-mean Gaussian. The pair $(C, A)$ is detectable. There are no uncontrollable modes of $\left(A, F Q_{w}^{1 / 2}\right)$ on the unit circle, where $Q_{w}^{1 / 2} \cdot\left(Q_{w}^{1 / 2}\right)^{T}$ is the covariance matrix of $w(k)$.

As long as the input-output transfer function of the system is the main interest, the model $(1,2)$ can be reformulated in the following innovation form [Verhaegen and Verdult, 2007].

$$
\begin{aligned}
\hat{x}(k+1) & =A \hat{x}(k)+B u(k)+K e(k), \\
y(k) & =C \hat{x}(k)+D u(k)+e(k) .
\end{aligned}
$$

Here, $K$ is the Kalman gain. The innovation $e(k)$ is white Gaussian due to the properties of $w(k)$ and $v(k)$. The covariance of $e(k)$, denoted by $\Sigma_{e}$, is determined by those of $w(k)$ and $v(k)$ [Ljung, 1987]. This type of model can be consistently identified from closed-loop data by the predictor-based subspace identification approach (PBSID) [Chiuso, 2007].

Since the innovation $e(k)$ is unknown, it can be replaced by $y(k)-C \hat{x}(k)-D u(k)$. A closed-loop observer thus results from $(3,4)$; i.e.

$$
\begin{aligned}
\hat{x}(k+1) & =\underbrace{(A-K C)}_{\Phi} \hat{x}(k)+(B-K D) u(k)+K y(k)(5) \\
y(k) & =C \hat{x}(k)+D u(k)+e(k) .
\end{aligned}
$$

We now define a data matrix for a signal $s \in\{x, u, y, e, z\}$, where $z(t) \triangleq\left[u^{T}(t) y^{T}(t)\right]^{T}$, as $\mathcal{S}_{t, p, N}$ equals $(\mathcal{S} \in$ $\{\mathcal{X}, \mathcal{U}, \mathcal{Y}, \mathcal{E}, \mathcal{Z}\})$

$$
\left[\begin{array}{cccc}
s(t) & s(t+1) & \cdots & s(t+N-1) \\
s(t+1) & s(t+2) & \cdots & s(t+N) \\
\vdots & \vdots & & \vdots \\
s(t+p-1) & s(t+p) & \cdots & s(t+p+N-2)
\end{array}\right] .
$$

The subscript $t$ indicates the time instant of the top left hand side element of $\mathcal{S}_{t, p, N}$; while $p$ and $N$ are respectively the number of block rows and columns of this matrix. The special case of a row block matrix $\mathcal{S}_{t, 1, N}$ will occur frequently.

Let $\mathcal{T}_{\star}^{L}$ denote the (block lower triangular) Toeplitz matrix with $L$ block columns and rows,

$$
\left[\begin{array}{cccc}
D_{\star} & & & \\
C B_{\star} & D_{\star} & & \\
\vdots & \vdots & \ddots & \\
C \Phi^{L-2} B_{\star} & C \Phi^{L-3} B_{\star} & \cdots & D_{\star}
\end{array}\right]
$$

where with " $\star$ " standing for " $z, u, y$ ", $B_{\star}$ respectively stands for " $[B-K D K], B-K D, K$ ", and $D_{\star}$ for "[ $D$ r 0$], D, 0 "$. "0" is a zero matrix of suitable dimension. For brevity, denote in the sequel $\mathfrak{B}=[B-K D K]$.

With these definitions, the model $(5,6)$ can be written on batch form as

$$
\begin{aligned}
\mathcal{Y}_{t, L, N}= & {\left[I_{L} \otimes\left(C \Phi^{p}\right)\right] \cdot \mathcal{X}_{t-p, L, N}+\mathcal{H}_{z}^{L, p} \cdot \mathcal{Z}_{t-p, p, N} } \\
& +\mathcal{T}_{z}^{L} \cdot \mathcal{Z}_{t, L, N}+\mathcal{E}_{t, L, N},
\end{aligned}
$$

Here, the symbol " $\otimes$ " stands for Kronecker product. $I_{L}$ is an $L \times L$ identity matrix. The block Hankel matrix, $\mathcal{H}_{z}^{L, p}$, is defined as

$$
\left[\begin{array}{cccccc}
C \Phi^{p-1} \mathfrak{B} & C \Phi^{p-2} \mathfrak{B} & \cdots & \cdots & \cdots & C \mathfrak{B} \\
0 & C \Phi^{p-1} \mathfrak{B} & \cdots & \cdots & \cdots & C \Phi \mathfrak{B} \\
\vdots & \vdots & & & & \vdots \\
0 & 0 & \cdots & C \Phi^{p-1} \mathfrak{B} & \cdots & C \Phi^{L-1} \mathfrak{B}
\end{array}\right]
$$

which contains $L$ block rows and $p$ block columns (the past horizon), where the higher order terms, $C \Phi^{p+j}, j \geq 0$, are replaced with zero matrices of appropriate dimension. The benefit of this treatment is that $\mathcal{H}_{z}^{L, p}$ only depends on the sequence of Markov parameters, $\left[C \Phi^{p-1} \mathfrak{B} \cdots C \mathfrak{B}\right]$.

The Markov parameters in $\mathcal{H}_{z}^{L, p}$ and $\mathcal{T}_{z}^{L}$ can be identified by solving the following data equation in a least-squares (LS) sense [Chiuso, 2007]; i.e. setting $L=1$.

$$
\mathcal{Y}_{t, 1, N}=\underbrace{C \Phi^{p} \mathcal{X}_{t-p, 1, N}}_{\mathbf{b}_{t-p, 1, N}}+\underbrace{\left[\begin{array}{ll}
\mathcal{H}_{z}^{1, p} & D
\end{array}\right]}_{\Xi} \cdot \underbrace{\left[\begin{array}{c}
\mathcal{Z}_{t-p, p, N} \\
\mathcal{U}_{t, 1, N}
\end{array}\right]}_{\mathbb{Z}_{i}}+\mathcal{E}_{t, 1, N}(.9)
$$

$\mathbb{Z}_{i}$ denotes the identification data matrix. Explicitly, $\Xi$ is the following block row vector,

$\left[C \Phi^{p-1}(B-K D), C \Phi^{p-1} K, \cdots, C(B-K D), C K, D\right]$. The LS estimate is then given by

$$
\hat{\Xi}=\mathcal{Y}_{t, 1, N} \cdot \mathbb{Z}_{i}^{\dagger}
$$

The term $\mathbf{b}_{t-p, 1, N}$ in the data equation will give rise to a bias that decays with $p$.

As a standard assumption in system identification, the data matrix $\mathbb{Z}_{i}$ has full row rank; i.e. $\exists \underline{\rho}_{z}>0$ such that

$$
\mathbb{Z}_{i} \mathbb{Z}_{i}^{T} \succeq \underline{\rho}_{z}^{2} I
$$


Here, for two matrices $\mathcal{M}_{1}$ and $\mathcal{M}_{2}, \mathcal{M}_{1} \succeq \mathcal{M}_{2}$ means that $\mathcal{M}_{1}-\mathcal{M}_{2}$ is positive semi-definite. Besides, we will use the following explicit bound on the covariance matrix of $\operatorname{vec}\left(\mathcal{X}_{t-p, 1, N}\right)$ in the discussions to follow; i.e. $\exists \bar{\sigma}_{x x}>0$ such that

$$
\left\|\operatorname{Cov}\left(\operatorname{vec}\left(\mathcal{X}_{t-p, 1, N}\right)\right)\right\|_{2} \leq \bar{\sigma}_{x x} .
$$

Treating $\Sigma_{e}$ as known, or using the estimate,

$$
\hat{\Sigma}_{e}=\operatorname{Cov}\left(\mathcal{Y}_{t, 1, N}-\hat{\Xi} \cdot \mathbb{Z}_{i}\right)
$$

in its place, is a standard practice in the statistical signal detection literature [Gustafsson, 2001, Kay, 1998]. We shall hence not distinguish between $\hat{\Sigma}_{e}$ and $\Sigma_{e}$ in the rest of the paper.

Similar to (8), an $L$-step output equation up to the current time instant $k$ can be constructed as

$$
\mathbf{y}_{k, L}=\mathbf{b}_{k, L}+\mathcal{H}_{z}^{L, p} \mathbf{z}_{k-L, p}+\mathcal{T}_{u}^{L} \mathbf{u}_{k, L}+\mathcal{T}_{y}^{L} \mathbf{y}_{k, L}+\mathbf{e}_{k, L} .
$$

Here, the noise covariance is denoted $\Sigma_{e}^{L}=I_{L} \otimes \Sigma_{e}$ and the bias term becomes

$\mathbf{b}_{k, L}^{T}=\left[\left(C \Phi^{p} \hat{x}(k-L-p+1)\right)^{T}, \cdots,\left(C \Phi^{p} \hat{x}(k-p)\right)^{T}\right]$. The residual generator is then a direct consequence of comparing the measured and computed outputs in the sliding window; i.e. with the true system parameters,

$$
\mathbf{r}_{k, L}=\left(I-\mathcal{T}_{y}^{L}\right) \mathbf{y}_{k, L}-\mathcal{H}_{z}^{L, p} \mathbf{z}_{k-L, p}-\mathcal{T}_{u}^{L} \mathbf{u}_{k, L} .
$$

If $L \leq p$, then $\Sigma_{e}$ and the identified parameters $\hat{\Xi}$ from (10) can fully parameterize the residual generator and the statistical fault detection test, with the unknown bias $\mathbf{b}_{k, L}$ ignored. We shall rewrite the residual generator built by the identified parameters as

$$
\mathbf{r}_{k, L}=\left(I-\overline{\mathcal{T}}_{y}^{L}\right) \mathbf{y}_{k, L}-\overline{\mathcal{H}}_{z}^{L, p} \mathbf{z}_{k-L, p}-\overline{\mathcal{T}}_{u}^{L} \mathbf{u}_{k, L} .
$$

\subsection{Robust data-driven FICSI detection}

The Markov parameters in $\overline{\mathcal{H}}_{z}^{L, p}, \overline{\mathcal{T}}_{y}^{L}, \overline{\mathcal{T}}_{u}^{L}$ contain errors Since the residual is a linear function of the estimated matrices, the residual again becomes Gaussian, whose distribution can be characterized as

$$
\mathbf{r}_{k, L} \sim\left\{\begin{array}{c}
\mathcal{N}\left(\mathbb{E} \mathbf{r}_{k, L}^{\text {no fault }}, \Sigma_{\Theta, e}^{L}\right), \text { fault free }, \\
\mathcal{N}\left(\mathbb{E} \mathbf{r}_{k, L}^{\text {faulty }}, \Sigma_{\Theta, e}^{L}\right), \text { faulty. }
\end{array}\right.
$$

The covariance $\Sigma_{\Theta, e}^{L}$ will be specified next. For the wellposedness of the problem, we need the following conditions.

Assumption 1. Both the nominal system and the system under the influence of additive faults are internally stable, i.e.

$$
\left\|\mathbb{Z}_{p, o l}\right\|_{2} \leq \bar{\rho}_{z, o l}, \forall k .
$$

Under this assumption, the state sequence,

$$
\hat{\boldsymbol{x}}_{k-p, L}=\left[\hat{x}^{T}(k-p-L+1) \cdots \hat{x}^{T}(k-p)\right]^{T},
$$

of the observer $(5,6)$ during the implementation of FICSI has a bounded covariance as (for simplicity, with the same bound $\bar{\sigma}_{x x}$ as in (12), without loss of generality),

$$
\left\|\operatorname{Cov}\left(\hat{\boldsymbol{x}}_{k-p, L}\right)\right\|_{2} \leq \bar{\sigma}_{x x}, \forall k .
$$

The key elements of the robustified FICSI design against parameter identification errors are summarized as follows.
- Let the signals in the identification experiment and fault detection respectively satisfy $(11,12)$ and $(17$, 18). If the past horizon $p$ is chosen such that

$$
\left\|C \Phi^{p}\right\|_{2}^{2}<\xi \cdot \frac{\left\|\Sigma_{e}\right\|_{2}}{\bar{\sigma}_{x x}^{2}}
$$

for an arbitrarily small positive number $\xi$; then

$$
\Sigma_{\Theta, e}^{L}=\left[\mathbb{Z}_{p, o l}^{T}\left(\mathbb{Z}_{i} \mathbb{Z}_{i}^{T}\right)^{-1} \mathbb{Z}_{p, o l}+I_{L}\right] \otimes \Sigma_{e}
$$

approximates the covariance matrix of the FICSI residual $\mathbf{r}_{k, L}$ computed by (15) in the following sense:

$$
\left\|\operatorname{Cov}\left(\mathbf{r}_{k, L}\right)-\Sigma_{\Theta, e}^{L}\right\|_{2}<\xi \cdot\left(1+\frac{\bar{\rho}_{z, o l}^{2}}{\underline{\rho}_{z}^{2}}\right) \cdot\left\|\Sigma_{e}\right\|_{2} .
$$

- Define the test statistic as

$$
\boldsymbol{t}_{k}=\left\|\left(\Sigma_{\Theta, e}^{L}\right)^{-\frac{1}{2}} \mathbf{r}_{k, L}\right\|_{2}^{2} .
$$

$\boldsymbol{t}_{k}$ has a noncentral $\chi^{2}$ distribution. However, if the past horizon $p$ also satisfies

$$
\left\|C \Phi^{p}\right\|_{2}^{2} \leq \frac{\zeta \cdot L \ell}{\left\|\Sigma_{e}^{-1 / 2}\right\|_{2}^{2}\left(\left\|\mathbb{E}\left[\operatorname{vec}\left(\mathcal{X}_{t-p, 1, N}\right)\right]\right\|_{2}+\left\|\mathbb{E} \hat{\boldsymbol{x}}_{k-p, L}\right\|_{2}\right)^{2}},
$$

for an arbitrarily small $\zeta>0$; then the cumulative distribution function (cdf) of $\boldsymbol{t}_{k}$ under the fault free case can be approximated by that of a central $\chi^{2}$ distribution.

With $p$ chosen satisfying (19) and (22), the robust FICSI method detects faults via comparing the statistics $\boldsymbol{t}_{k}$ in (21) with the threshold, $\gamma_{\chi_{L \ell}^{2}, \alpha}$, determined by the central $\chi^{2}$ distribution with a DoF of $L \ell$, i.e. $\chi_{L \ell}^{2}$, and a prespecified false alarm rate (FAR), $\alpha$.

\section{DATA DRIVEN FAULT ISOLATION}

Safety-critical systems usually contain redundancies in their hardware components. With the availability of three sensors for each output channel, a majority-vote-based scheme can be implemented, without using any FDI algorithms. However in the case of only two sensors for each output channel, as is the case of this wind turbine benchmark, no majority exists for each output. One then has to rely on software redundancy, i.e. an FDI algorithm. A bank of robust FICSI detection filters shall thus be designed and implemented in parallel, each with a different set of sensors.

Let $S_{i}^{1}, S_{i}^{2}$ respectively denote the two sensors measuring the $i$-th output channel. Since there are $\ell$ output channels in total, one shall run $2^{\ell}$ robust FICSI detection filters in parallel. Denote these filters by $\mathcal{F}_{j} \mid\left\{S_{1}^{i_{1}}, \cdots, S_{\ell}^{i_{\ell}}\right\}, j=$ $1, \cdots, 2^{\ell}, i_{1}, \cdots, i_{\ell} \in\{1,2\}$. Let $\mathbb{I}_{q f}$ denote the set of sensor indices contained in all the quiet filters, which do not give a fault alarm at time instant $k$. Denote the indices contained in the complimentary set of $\mathbb{I}_{q f}$ by $\overline{\mathbb{I}_{q f}}$.

Theorem 1. Assume there are more than one sensors measuring each individual output channel in a plant; and the sensors measuring the same output do not fail at the same time. The sensor indices are contained in the set $\overline{\mathbb{I}_{q f}}$ if and only if their corresponding sensors are faulty.

Proof The "if' part is obvious by the definition of $\overline{\mathbb{I}_{q f}}$. We prove the "only if" part by contradiction. Assume $\exists S_{j}^{i_{j}} \in \overline{\mathbb{I}_{q f}}$ for the $j$-th output channel, which is fault free. 
Since the sensor $S_{j}^{i_{j}}$ has no fault, and not all sensors measuring the same channel can simultaneously fail, there exists a filter containing $S_{j}^{i_{j}}$, i.e. $\mathcal{F}_{\star} \mid\left\{\cdots, S_{j}^{i_{j}}, \cdots\right\}$, which does not fire an alarm. This indicates that all the sensor indices contained in $\mathcal{F}_{\star} \mid\left\{\cdots, S_{j}^{i_{j}}, \cdots\right\}$ belong to the set of $\mathbb{I}_{q f}$, including $S_{j}^{i_{j}}$. This contradicts with the assumption that $S_{j}^{i_{j}} \in \overline{\mathbb{I}_{q f}}$.

For instance, if $\ell=2$, one needs 4 parallel filters. The following table shows the alarms of these filters (denoted by a "Ф") under different individual sensor faults, each occurring at a different time instant.

\begin{tabular}{|c|c|c|c|c|}
\hline & $\bar{S}_{1}^{1}$ & $\overline{\bar{S}_{1}^{2}}$ & 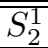 & 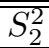 \\
\hline $\begin{array}{l}\mathcal{F}_{1} \mid\left\{S_{1}^{1}, S_{2}^{1}\right\} \\
\mathcal{F}_{2} \mid\left\{S_{1}^{1}, S_{2}^{2}\right\} \\
\mathcal{F}_{3} \mid\left\{S_{1}^{2}, S_{2}^{1}\right\} \\
\mathcal{F}_{4} \mid\left\{S_{1}^{2}, S_{2}^{2}\right\} \\
\end{array}$ & $\begin{array}{l}\text { बा } \\
\text { ब }\end{array}$ & ब & ๑ & ब \\
\hline
\end{tabular}

For example, along the first column, $\mathbb{I}_{q f}=\left\{S_{1}^{2}, S_{2}^{1}, S_{2}^{2}\right\}$, and hence $\overline{\mathbb{I}_{q f}}=S_{1}^{1}$, which identifies the faulty sensor. On the other hand, if $S_{1}^{1}, S_{2}^{1}$ fail at the same time, then only the filter $\mathcal{F}_{4}$ is quiet. Hence, $\overline{\mathbb{I}_{q f}}=\left\{S_{1}^{1}, S_{2}^{1}\right\}$. However, if both $S_{i}^{1}, S_{i}^{2}, \forall i=1, \cdots, \ell$ measuring the same $i$-th output channel fail at the same time, then this logic cannot isolate faults, since all the parallel filters will give an alarm simultaneously.

\section{FDI OF THE WIND TURBINE BENCHMARK}

\subsection{Benchmark description}

The benchmark is a three-bladed, horizontal-axal, and variable-speed wind turbine with a full converter. It is controlled in closed loop, to follow the power reference, or if not possible to minimize the reference error. The control inputs are the blade pitch angle reference $\beta_{r}$ and the generator torque reference $\tau_{g, r}$. The measured quantities are the pitch angles of the three blades $\beta_{1}, \beta_{2}, \beta_{3}$, rotor speed $\omega_{r}$, and generator speed $\omega_{g}$. Two sensors are available for each output channel, respectively denoted with an additional subscript " $m 1$ " and " $m 2$ " in $\beta_{1}, \beta_{2}, \beta_{3}, \omega_{r}, \omega_{g}$; e.g. $\beta_{1, m 1}, \omega_{r, m 2}$. The turbine is disturbed by unknown wind speed $V_{w}$, whose profile was measured from a wind park. See Fig. 1. The closed-loop control scheme is shown in Fig. 2. The sampling frequency is $100 \mathrm{~Hz}$.

The benchmark turbine is subject to no more than one single fault at each sampling instant. Eight different faults are defined along the time span of 4400 seconds, including sensor, actuator, and plant faults. These faults and their durations are defined in details in [Odgaard et al., 2009]. To avoid repetition, we shall only list the three sensor faults that we especially considered in this paper, as listed in following table. The onset of the other faults defined in the benchmark can also be seen in Fig. 4 .

\begin{tabular}{r|ll}
\hline \hline$\#$ & fault & duration \\
\hline 1 & $\beta_{1, m 1}$ fixed to $5^{\circ}$ & $2000 \sim 2100 \mathrm{sec}$ \\
3 & $\beta_{3, m 1}$ fixed to $10^{\circ}$ & $2600 \sim 2700 \mathrm{sec}$ \\
4 & $\omega_{r, m 1}$ fixed to $1.4 \mathrm{rad} / \mathrm{s}$ & $1500 \sim 1600 \mathrm{sec}$ \\
\hline \hline
\end{tabular}

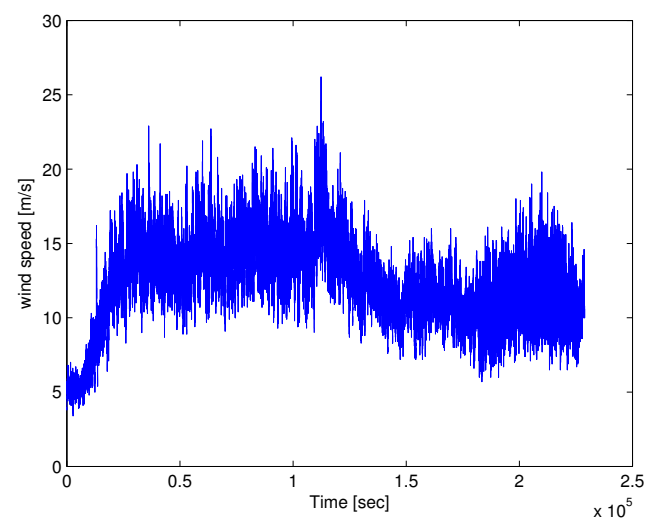

Fig. 1. The real measured wind speed profile used in the benchmark [Odgaard et al., 2009].

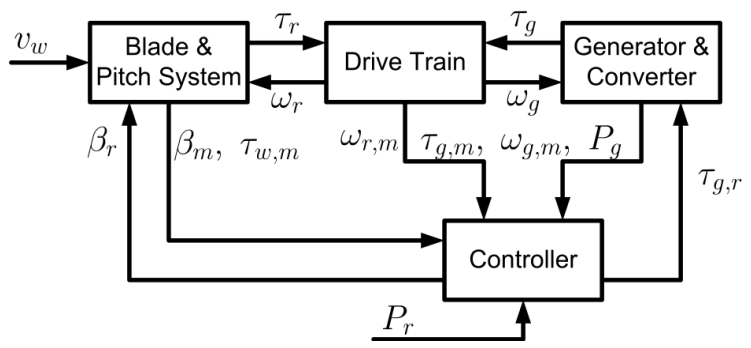

Fig. 2. System overview of the wind turbine benchmark model [Odgaard et al., 2009].

The requirements for FDI schemes by the benchmark include the time of detection no longer than 10 sampling instants, no missed detections, and mean time between false detection no larger than $10^{5}$ sampling instants.

\subsection{Data-driven FDI design}

The variance of the measurement noise in the sensors, denoted as $\sigma_{\star}^{2}$, is respectively defined in the benchmark as follows.

$$
\begin{aligned}
& \sigma_{\beta_{1, m 1}}^{2}=\sigma_{\beta_{1, m 2}}^{2}=\sigma_{\beta_{2, m 1}}^{2}=\sigma_{\beta_{2, m 2}}^{2}=\sigma_{\beta_{3, m 1}}^{2}=\sigma_{\beta_{3, m 2}}^{2}=0.2 \\
& \sigma_{\omega_{g, m 1}}^{2}=\sigma_{\omega_{g, m 2}}^{2}=0.05, \sigma_{\omega_{r, m 1}}^{2}=\sigma_{\omega_{r, m 2}}^{2}=0.0251 .
\end{aligned}
$$

Due to the strong unknown wind disturbances and the high noise level in the sensors, we chose to apply the robust FICSI detection design reviewed in Sec. 2.2. On the other hand, we turned to the isolation logic proposed in Sec. 3 , due to the availability of hardware redundancies in the benchmark. The FDI scheme is illustrated in Fig. 3.

To obtain the robust FICSI detection filter parameters, the identification experiments were designed as follows. The wind speed profile was set to the mean of the real measured wind data as shown in Fig. 1, i.e. D_v_wind $\equiv 12.3$ in the SimuLink model, "BenchMark.mdl". The sophisticated wind model in this SimuLink file was not at all changed. We also emphasize that the wind speed was not used in solving the LS problem. No extra excitation signals were added to this model. We only used the data from the first 200 seconds, i.e. $N=2 \times 10^{4}$. The horizons were chosen as $p=60, L=50$. The FAR was set to 0.001. Note that since the pitch angles of the three blades were identical with each other, only one of them could be included in the identification data for the well-posedness of 


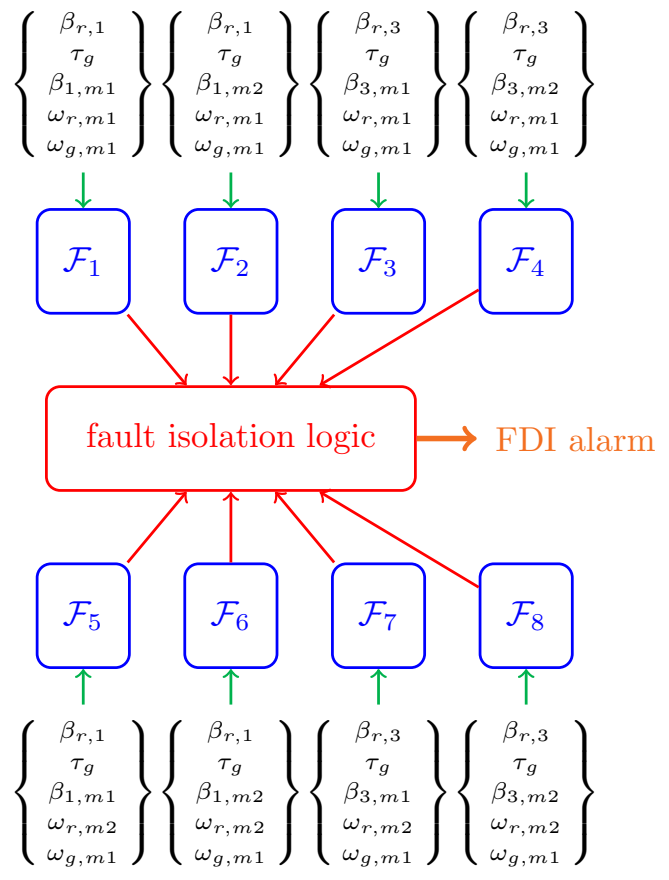

Fig. 3. Robust FICSI detection filter bank.

the LS solution. We only selected three output channels for the identification, i.e. $\beta_{1, m 1}, \omega_{r, m 1}, \omega_{g, m 1}$. The two input channels are $\beta_{r}, \tau_{g}$. The estimated $\Sigma_{e}$ equals

$$
\left[\begin{array}{ccc}
0.1980 & -0.0001 & -0.0005 \\
-0.0001 & 0.0533 & -0.0002 \\
-0.0005 & -0.0002 & 0.0246
\end{array}\right],
$$

which are good estimates to the true values, implying that the identification results are satisfactory. The same set of parameters were then used to implement a bank of 8 filters, with different sets of I/O signals, as illustrated in Fig. 3.

\subsection{Simulation results}

We then implemented the designed filter bank to the SimuLink model, "FDIBenchMark.mdl", where faults were injected according to their definitions. It should be noticed that the control scheme in "FDIBenchMark.mdl" is different from that in "BenchMark.mdl"; i.e. the control signal $\beta_{r}$ to each blade is further corrected with the tracking error of each blade:

$$
\beta_{r, i}=\beta_{r}+\underbrace{\beta_{i}-\left(\beta_{i, m 1}+\beta_{i, m 2}\right) / 2}, i=1,2,3,
$$

where $\beta_{i}$ is the pitch angle of the $\mathrm{i}$-th blade. However, the underbraced term is noisy, and hence increases the noise level of the control signal $\beta_{r, i}$. For instance, if $\beta_{i, m 1}, \beta_{i, m 2}$ are correct measurements, then the underbraced term is $-\left(e_{\beta_{i, m 1}}+e_{\beta_{i, m 2}}\right) / 2$; i.e. the sum of the two sensing noise. In our experiments, we turned the underbraced compensation term to zero to keep the characteristics of the control signals unchanged from the identification model to the fault-injection model. The rest of "FDIBenchMark.mdl" was not at all modified.

The outputs of $\mathcal{F}_{3} \mid\left\{\beta_{r, 3}, \tau_{g}, \beta_{3, m 1}, \omega_{r, m 1}, \omega_{g, m 1}\right\}$ in this filter bank was plotted in Fig. 4 for the entire 4400 seconds of simulation. Obviously, the filter was especially sensitive to Faults $\# 3$ and \#4. Except the plant fault $\omega_{n 3}, \zeta_{n 3}$, the

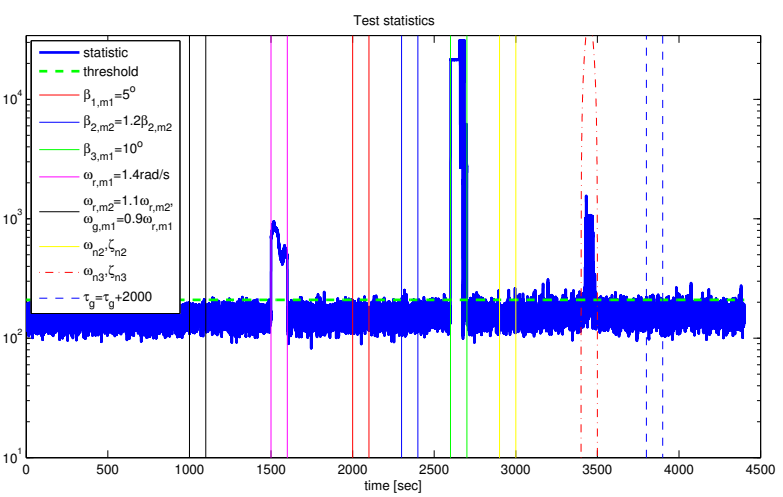

Fig. 4. Robust FICSI detection statistics of filter $\mathcal{F}_{3} \mid\left\{\beta_{r, 3}, \tau_{g}, \beta_{3, m 1}, \omega_{r, m 1}, \omega_{g, m 1}\right\}$.

filter had no response to the other faults. The reason can be attributed to their small fault amplitudes as compared to both the measurement noise and the true output signals. Besides, due to the nonlinear dynamics and the nonGaussian noise, i.e. the wind disturbances, spikes in the test statistics appeared from time to time, violating the theoretical threshold. However, it is also clear that the average of the statistics stayed below the threshold, when the faults were absent. This motivated us to smoothen the statistics [Qin and Li, 2001], by

$$
\tilde{\boldsymbol{t}}_{k}=\gamma \cdot \tilde{\boldsymbol{t}}_{k-1}+(1-\gamma) \cdot \boldsymbol{t}_{k}
$$

where $\gamma$ was chosen as 0.99 . No spikes, i.e. false alarms, appeared again in the smoothed outputs $\tilde{\boldsymbol{t}}_{k}$ of $\mathcal{F}_{3}$, as shown in Fig. 5. Besides, neither detection delay, nor missed detection, was found in this experiment. The requirements in the benchmark problem were therefore satisfied. We only showed the three sensor faults that were significant enough to be detected. For clarity, the time horizon was shortened to 3000 seconds.

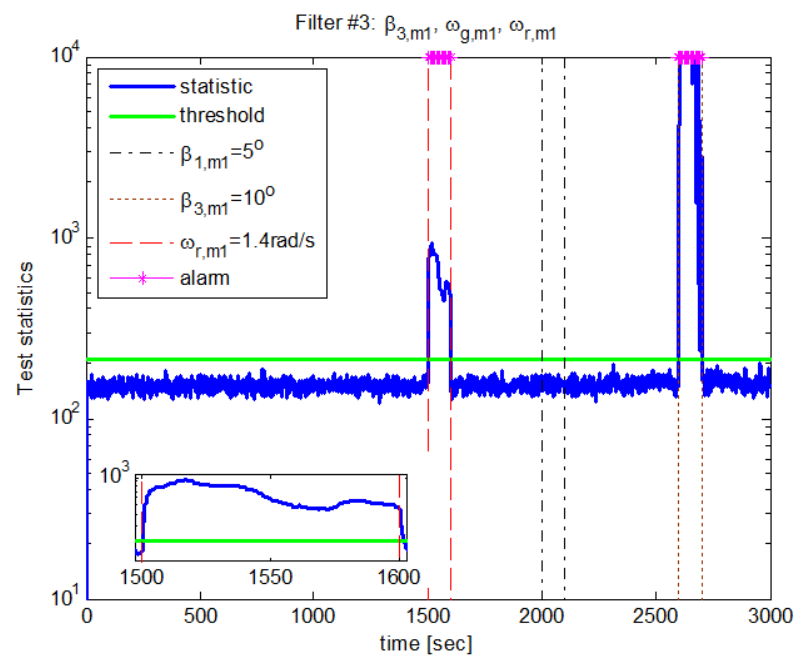

Fig. 5. Smoothed test statistics of filter $\mathcal{F}_{3}$.

All the eight filters as depicted in Fig. 3 were smoothed by the low pass filter (23). Neither detection delay, nor missed detection, was discovered in any of the filters. The results are plotted in Fig. 6. The fault isolation logic is verified in the following table, where for brevity, the sensor sets 

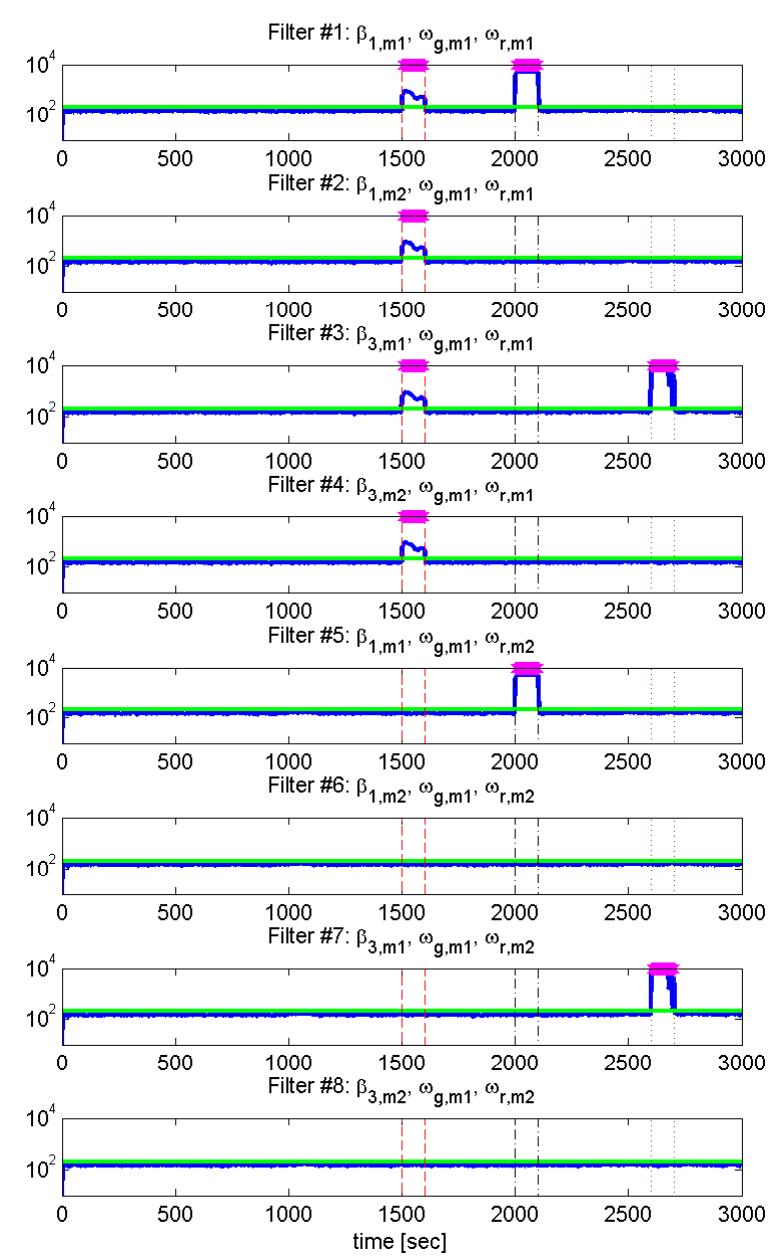

Fig. 6. Statistics of filter outputs in the fault isolation scheme of Fig. 3. Solid blue: statistics. Solid green: threshold. Dash-dotted black: Fault \#1. Dotted brown: Fault \#3. Dashed red: Fault \#4. Purple stars: alarms.

to each filter are omitted; and $\emptyset$ stands for empty set. Obviously, the three sensor faults were correctly located.

\begin{tabular}{|c|c|c|c|c|c|c|}
\hline & $\beta_{1, m 1}$ & $\beta_{1, m 2}$ & $\beta_{3, m 1}$ & $\beta_{3, m 2}$ & $\omega_{r, m 1}$ & $\omega_{r, m 2}$ \\
\hline $\mathcal{F}_{1}$ & 9 & & & & 9 & \\
\hline $\mathcal{F}_{2}$ & & & & & 9 & \\
\hline $\mathcal{F}_{3}$ & & & ब & & $\overline{9}$ & \\
\hline $\mathcal{F}_{4}$ & & & & & $\frac{1}{9}$ & \\
\hline $\mathcal{F}_{5}$ & ब & & & & & \\
\hline $\mathcal{F}_{6}$ & & & & & & \\
\hline $\mathcal{F}_{7}$ & & & ब & & & \\
\hline $\mathcal{F}_{8}$ & & & & & & \\
\hline$\overline{\overline{\mathbb{I}_{q f}}}$ & $\overline{\beta_{1, m 1}}$ & $\emptyset$ & $\overline{\beta_{3, m 1}}$ & $\bar{\emptyset}$ & $\overline{\omega_{r, m 1}}$ & $\bar{\emptyset}$ \\
\hline
\end{tabular}

\section{CONCLUSIONS}

In this paper, we have investigated a robustified datadriven fault detection algorithm on a wind turbine benchmark problem. We have also developed a hardware redundancy based fault isolation scheme, especially for this robustified detection filter design, where no projection onto fault sensitive subspaces is allowed. The simulation results have verified the effectiveness of these methods on this challenging benchmark problem. Future work is to incorporate this FDI scheme with controller reconfiguration.

\section{REFERENCES}

M. Basseville and I.V. Nikiforov. Detection of Abrupt Changes - Theory and Application. Prentice Hall, 1993.

P. Caselitz, J. Giebhardt, M. Mevenkamp, and M. Reichardt. Application of condition monitoring systems in wind energy converters. In the Proceedings of the EWEC. Dublin, 1997.

J. Chen and R.J. Patton. Robust Model-Based Fault Diagnosis for Dynamic Systems. Kluwer Academic Publishers, 1999.

A. Chiuso. On the relation between CCA and predictorbased subspace identification. IEEE Transactions on Automatic Control, 52:1795-1812, 2007.

S.X. Ding. Model-based Fault Diagnosis Techniques Design Schemes, Algorithms, and Tools. Springer, 2008.

S.X. Ding, P. Zhang, A. Naik, E.L. Ding, and B. Huang. Subspace method aided data-driven design of fault detection and isolation systems. Journal of Process Control, 19:1496-1510, 2009.

J. Dong, B. Kulcsár, and M. Verhaegen. Subspace based fault identification for LPV systems. In the Proceedings of the 7th IFAC SafeProcess, pages 335-341. Barcelona, Spain, 2009.

J. Dong, M. Verhaegen, and F. Gustafsson. Data driven fault detection with robustness to uncertain parameters identified in closed loop. In Proceedings of the 49th IEEE $C D C$, pages 750-755. Atlanta, USA, 2010.

J. Dong, M. Verhaegen, and F. Gustafsson. Statistical fault detection with robustness to uncertain parameters identified from closed loop data. Automatica, Provisionally accepted, 2011.

F. Gustafsson. Adaptive filtering and change detection. John Wiley \& Sons, 2001.

S.M. Kay. Fundamentals of Statistical Signal Processing: Detection Theory. Prentice-Hall, 1998.

L. Ljung. System Identification - Theory for the User. Prentice-Hall, Englewood Cliffs, 1987.

P.F. Odgaard, J. Stoustrup, and M. Kinnaert. Fault tolerant control of wind turbines a benchmark model. In the Proceedings of the 7th IFAC SafeProcess, pages 155-160. Barcelona, Spain, 2009.

S.J. Qin and W. Li. Detection and identification of faulty sensors in dynamic processes. AIChE Journal, 47:1581$1593,2001$.

T.G. van Engelen. Control design based on aero-hydroservo-elastic linear models from TURBU (ECN). In the Proceedings of EWEC. Milan, Italy, 2007.

M. Verhaegen and V. Verdult. Filtering and System Identification: A Least Squares Approach. Cambridge University Press, 2007.

X. Wei and M. Verhaegen. Fault detection of large scale wind turbine systems: A mixed $\mathcal{H}_{\infty} / \mathcal{H}_{-}$index observer approach. In the Proceedings of 16th Mediterranean Conference on Control and Automation, pages 16751680. Ajaccio, France, 2008.

X. Wei, M. Verhaegen, and T. van den Engelen. Sensor fault detection and isolation for wind turbines based on subspace identifcation and kalman filter techniques. International Journal of Adaptive Control and Signal Processing., 24:687-707, 2010. 